\title{
AC 2012-4161: A WIRELESS SENSOR NODE POWERED BY SOLAR HAR- VESTER FOR MARINE ENVIRONMENT MONITORING AS A SENIOR DESIGN PROJECT
}

\author{
Dr. Radian G. Belu, Drexel University
}

Radian Belu is Assistant Professor within the Engineering Technology (ET) program at Drexel University, Philadelphia, USA. He is holding the second position as Research Assistant Professor at Desert Research Institute, Renewable Energy Center, Reno, Nev. Before joining Drexel University, Belu held faculty and research positions at universities and research institutes in Romania, Canada, and the United States. He also worked for several years in industry as a project manager and senior consultant. He has taught and developed undergraduate and graduate courses in electronics, power systems, control and power electronics, electric machines, instrumentation, radar and remote sensing, numerical methods and data analysis, space and atmosphere physics, and physics. His research interests included power system stability, control and protection, renewable energy system analysis, assessment and design, power electronics and electric machines for wind energy conversion, radar and remote sensing, wave and turbulence simulation, measurement and modeling, numerical modeling, electromagnetic compatibility, and engineering education. During his career, Belu published several papers in referred journals and in conference proceedings in his areas of the research interests. He has also been PI or Co-PI for various research projects in the United States and abroad in power systems analysis and protection, load and energy demand forecasting and analysis, renewable energy analysis, assessment and design, turbulence and wave propagation, radar and remote sensing, instrumentation, atmosphere physics, electromagnetic compatibility, and engineering education.

\section{Dr. Irina Nicoleta Ciobanescu Husanu, Drexel University}

Irina Ciobanescu Husanu, Co-PI, is Assistant Professor in applied engineering at Drexel University. She received her Ph.D. degree in mechanical engineering from Drexel University and also a M.S. degree in aeronautical engineering. Her research interest is in thermo-fluid sciences with applications in microcombustion, fuel cells, green fuels, and plasma-assisted combustion. Husanu has prior industrial experience in aerospace engineering that encompasses both theoretical analysis and experimental investigations, such as designing and testing of propulsion systems including design and development of pilot testing facility, mechanical instrumentation, and industrial applications of aircraft engines. Also, in the past seven years she gained experience in teaching M.E. and E.T. courses in thermal-fluid and energy conversion areas from various levels of instruction and addressed to a broad spectrum of students, from freshmen to seniors, from high school graduates to adult learners. She also has extended experience in curriculum development.

\section{Ms. Dunia Tania Periverzov}

(C)American Society for Engineering Education, 2012 


\title{
A Wireless Sensor Node Powered by Solar Harvester for Marine Environment Monitoring as a Senior Design Project
}

\begin{abstract}
Improving the design component in undergraduate engineering education has been an immediate and pressing concern for educators, professional societies, industrial employers and agencies concerned with national productivity and competitiveness. The projects are a valuable component of the science and engineering education. The design experience develops the students' lifelong learning skills, self-evaluations, self-discovery, and peer instruction in the design's creation, critique, and justification. Students learn to understand and make use of the manufacturer data sheets, application notes, and technical manuals when developing their design projects. The experience, which would be difficult to complete individually, gives the students a sense of satisfaction and the accomplishment that is often lacking in many engineering courses, using traditional teaching approaches. Furthermore, the design experience motivates student learning and develops skills required in industry. This paper discusses the development of a student project involving a number of senior undergraduate students at our engineering technology program. A simple, low cost solar power system to power a wireless sensor network (WSN) was developed during this project. The proposed WSN may be used for monitoring a coastal shallow water marine environment. It is composed of several sensor nodes or buoys. The description of this harvester, the system characteristics and performances are presented in details. Various aspects of the educational experience are examined such as the educational goals of the project, project organization, and outcomes ${ }^{1-3}$. Innovative educational approaches are described such as brainstorming session and discussion with students of high-level choices described by a decision tree, component selections, simulations and system performance and characteristics computation. In the second part of the paper the design solution that was adopted is described in details. The adopted design solution includes: power electronics circuitry (DC-DC converter design and test), maximum power point tracking (MPPT) algorithms, control strategies, battery and super-capacitor selection as energy buffers, and overall system performances. Different MPPT and charging algorithms were analyzed and evaluated for their effectiveness in solar energy conversion system, as well as the control algorithms and implementation to maximize the power output. The project is a good example of multi-disciplinary cooperation as well as providing valuable hands-on experience. In addition to providing useful lessons in teamwork and project management, the project will provide a working demonstration wireless sensor network and solar energy system. The goal of the design project is to explore and enhance students understanding of the fundamental engineering principles, power circuit simulation capability and hands-on demonstration of system prototyping.
\end{abstract}

\section{Introduction and Project Rationale}

The wireless sensor networks (WSNs) is an autonomous network system which consists of large number of micro sensor nodes and has the characteristics of capability of sensing, calculation communication and low cost, and low power. It is a "smart" system that can accomplish various monitoring tasks, according to different environment conditions. Monitoring of water environment is one of its typical applications. Compared with existing real-time automatic water 
environment monitoring systems, WSNs-based water environment monitoring system has strongpoint as follows ${ }^{3,5-8}: 1$ ) Less effect of the system on ecological environment: nodes transmit water environment parameters to base station by low power and low radiation wireless channel and multi-hop communication protocol. Marine wireless sensor networks offer an unmatched option to a wide range of different domains. The significance of the aforementioned research lies in the fact that it opens the door for a variety of applications as well as new areas of relevant research in wireless networks. The possibility of having hundreds of thousands of sensor nodes diving in the ocean collecting data about the different inhabitants offers a unique opportunity for ocean studies and researchers in the field. The ability to seed wireless sensors that can dive deep in the ocean taking real-time pictures and reporting relevant data about the oceanic life can play a major role in bringing ocean research to new levels. In the following we present the development and designed of a solar energy harvester that can be used to provide power to a WSN for marine environment monitoring system. Coastal marine systems are particularly vulnerable to the effects of human activity attendant on industrial, tourist and urban development. Information and communications technologies offer new solutions for monitoring such ecosystems in real time. Therefore, during the past decade various initiatives emerged, from small-scale networks to complex coastal observation systems. Among small-scale networks, WSNs are a highly attractive solution for its easiness in deployment, operating and dismantling. WSNs are also relatively inexpensive.

Energy harvesting or the process of acquiring energy from the surrounding environment has been a continuous human endeavor throughout history, e.g. the use of watermills in ancient Greece, and of sailboats by Phoenicians and Egyptians, circa 4000 B.C. Unlike the conventional electric power generation systems, in energy harvesting concept, fossil fuels are not used and the generation units might be decentralized. There are many sources for harvesting energy. Solar, wind, ocean, hydro, electromagnetic, electrostatic, thermal, vibration, and human body motion are renewable sources of energy. Even the energy of radio frequency waves, propagated due to television and radio broadcasting in the environment, can be harvested. Economic, environmental, and geopolitical constraints on global conventional energy resources started forcing the nations to accelerate energy harvesting from renewable energy sources. Thus, advanced technical methods should be developed to increase the efficiency of devices in harvesting energy from various environmentally friendly resources and converting them into electrical energy. These developments have sparked the interest in engineering community as well as in the engineering education community to develop more energy harvesting applications and new curriculums for renewable energy and energy harvesting topics. Nowadays, there is an increasing interest to harvest energy at a much smaller scale, for applications such as the ones found in many embedded systems where the power requirements are often small (less than 100 $\mathrm{mW}$ ). Sustaining the power requirement for autonomous wireless and portable devices is an important issue. However, this progress has not been able to keep up with the development of microprocessors, memory storage, and wireless technology applications. For example, in wireless sensor networks, battery-powered sensors and modules are expected to last for a long period of time. However, conducting battery maintenance for a large-scale network consisting of hundreds or even thousands of sensor nodes may be difficult, if not impossible. Ambient power sources, as a replacement for batteries, come into consideration to minimize the maintenance and the cost of operation. Power scavenging may enable wireless and portable electronic devices to be completely self-sustaining, so that battery maintenance can be eventually removed. When 
compared with energy stored in common storage elements, such as batteries, capacitors, and the like, the environment represents a relatively infinite source of available energy. Systems continue to become smaller, yet less energy is available on board, leading to a short runtime for a device or battery life. Researchers continue to build high-energy density batteries, but the amount of energy available in the batteries is not only finite but also low, which limits the life time of the systems. Extended life of the electronic devices is very important; it also has more advantages in systems with limited accessibility, such as those used in monitoring a machine or an instrument in a manufacturing plant used to organize a chemical process in a hazardous environment. The critical long-term solution should therefore be independent of the limited energy available during the functioning or operating of such devices.

\subsection{Objectives of Project Work}

In response to these demands, universities offering baccalaureate and graduate degrees in electrical engineering or engineering technology must develop curricula to educate a workforce that is well equipped to meet these challenges. Unfortunately, US universities have not kept pace with the growing fields of power electronics, wireless networks or renewable energy, and they are not educating enough students in these recent technology applications, as needed by our industries. Rather this growth has been principally research and industry oriented. Little progress is being reported, with very few notable exceptions on the role of educational institutions in either keeping pace with this growth or addressing the importance of introducing new emerging engineering technologies, applications, and effective classroom and laboratory instruction. Engineering and engineering technology programs must offer a relevant and validated curriculum that prepares students for post-graduation success. Courses that cover traditional subject matter in mathematics, the sciences, engineering economics and other related topics provide the foundation of knowledge upon which specific skill sets are added. However, it is critical for engineering/technology to transition from theoretical work in the classroom towards experiential learning with applications of technology and design ${ }^{1-3}$. The main objective of senior design courses in engineering and engineering technology curricula is to bridge the gap between academic theory and real world practice. Accordingly, the proposed senior projects should include elements of both credible analysis and experimental proofing as discussed in ABETs criteria ${ }^{5}$. The senior design project can serve as an excellent culminating experience in the program of study when it focuses on research and design projects that have practical value to consumers or to industry. For the ET program at our university, the senior design course is a year-long educational journey (three quarters) that takes an idea generated by a student or an industrial sponsor and culminates in a product or project. This course is an excellent capstone experience, which requires both teamwork and individual skills in solving a modern industrial problem $^{6-10}$. Senior design projects seminars in fall and spring quarters bring the students, faculty, and industrial partners together to see the student's results and to give them the additional experience of public presentation of their work.

The importance of project work in the curriculum of our undergraduate engineering programs is well understood. Students and faculty need to understand the objectives of doing project work, to have mechanisms for selection of attractive topics, focus and scope of work, estimation of efforts required and facilities needed, etc. Students want and need to know the best practices also. What is the focus of engineering educations is an important question that needs to be asked and 
introspected upon frequently by all of us. An engineer is a technical problem solver - designing large systems like road / power / gas / water / communications networks or large industries / utilities; building large systems like power plants, dams, server banks, etc.; operating large engineering systems like communications services, wide area networks, power systems, etc.; or managing systems and services. Therefore, an engineer should be a designer, thinker, innovator and systems integrator. Hence, the educational system should inculcate into its students various aspects such as engineering principles, standards and practices, design methodologies, modeling and optimization capabilities, systems analysis and integration techniques and new technologies and research areas. An engineer can become a thinker, a creative person or an innovator only if he is allowed to independently put together all aspects of learning to solve a practical problem and look at alternatives. Lectures, tutorials and laboratories allow students to acquire engineering and scientific knowledge. However, only project work gives them the opportunity to become a problem solver or an innovator. Hence, project work is a very important constituent of our engineering curriculum. It is necessary to understand the role and importance of project work in the professional world. Project work provides several opportunities to students to learn several aspects of importance to an engineer that cannot be taught in a class room or laboratory.

For an engineer in industry, a project is a sequence of tasks required to reach an objective. Typically, the objective is to design a device or process that has value to a customer (user). The project begins by defining a performance problem associated with an application and ends with a design solution. The problem drives the learning required to complete the project. Managing the project requires the engineer to demonstrate effective teamwork, clear communication and the ability to balance the social, economic and environmental impacts of the project. Project-based learning (PBL) is based on the practice of solving problems. The concept of problem-based learning was first developed in the medical field in the mid-1950's. It has since been adopted in a variety of educational disciplines ${ }^{1-4}$. Traditionally, the educational process involves students first learning the fundamentals and then utilizing "total recall" to apply these facts to solve a problem; learning objectives are set by the instructor and principles are presented to the students through lectures. Assignments are given to reinforce the application of the concepts, but often students merely "learn" what is necessary to pass the test or "repeat-back" information to satisfy the instructor. In contrast, the PBL approach employs a problem as the driving force for learning the fundamental principles that are required to find a solution. Moreover, this approach provides a context that makes learning the fundamentals more relevant and, hence, results in better retention by students ${ }^{1-3}$. For clarity, we view problem-based learning as pertaining to the development of knowledge based on the fundamental principles of science and mathematics and project-based learning to include mastering the engineering skills required to implement a design solution.

The purpose of this paper is to describe the energy harvesting project involved in our capstone senior design project. The lessons learned are presented and the ways to improve project management are discussed. Our senior design project course is a 3-term core course usually taken by the students during their terminal year in the ET program.

\section{Project Overview, Goals, Constraints and Solutions and Design Content}

This project is an underway project for MET 421/422/423 (Senior Project Design), preformed by a team of four students, two electrical and two mechanical seniors. MET 421/422/423 is a 
sequence of three-quarter capstone project design courses required for all the BSET majors. The course focuses on planning, development, and implementation of an engineering design project, which includes formal report writing, project documentation, group presentations, and project demonstrations. The goal of these courses is to demonstrate the ability to manage a major project involving the design and implementation of products with a mixture of electrical and mechanical elements as a member of a product development team. In these project-based courses, the students are expected to effectively manage their time and team efforts to produce a finished product in three ten-week quarters. No textbook is required. Progress and formal reports, and oral presentations constitute integral components of this course sequence. Before beginning the projects, student teams are provided adequate training in project formulation and resource analysis, performance goals and team expectations, public presentations of project work, and individual project supervision ${ }^{9-11}$.

ABET defines Engineering Design as: "The process of devising a system, component, or process to meet the desired needs ${ }^{10,11}$. It is a decision making process, in which the basic sciences, mathematics, and engineering sciences are applied to optimally convert resources to meet stated objective $^{1}$. The fundamental elements of the design process are: the establishment of the objectives and criteria, synthesis, analysis, construction, testing and evaluation". In our senior design classes we have placed this definition at the core of our courses. First we focus on objectives and ask the student to write a short proposal stating these objectives, principles, and the decisive factors to reach the stated goals. These projects involved elements of structural design, wind and solar energy resource assessment, electrical, electronics and computer engineering system design. The second step is conceptualization and laying down how to achieve the stated objectives. At this junction the students are encouraged to draw a block diagram showing different components of the system they want to design. A set of questions are posed to students to further understand the task at hand. These are typical questions:

- What are the inputs to the system and, what are their characteristics and magnitudes?

- Do the inputs require conditioning?

- What is the medium through which inputs are interfaced to the system under consideration?

- Do the inputs dictate to the system to be designed how to behave, or just activates the system?

- What is the voltage, current and power requirements for the load?

- Is it a single output or multi-output system?

- Are there feedback loops in the system?

- Do the loads require separate power supplies?

Once the students compile the answers to these questions, they are directed to perform system analysis, design, component purchase and fabrication, building and testing of the prototype, as well as the overall design improvements.

The harvester must reliably and autonomously operate for ten years after installation with no maintenance while capturing enough energy to power the communication end node of the sensor network. Though the power consumption of the end node was not fully defined, it was assumed to be the power consumption of its main component: the sensors used to measure atmospheric and marine parameters, such: air pressure, temperature, and humidity, sea temperature, etc. The 
harvester also needs an energy storage device to store excess energy and use it to power the load when the harvester does not produce power. It must be resistant to the elements, wildlife, and others affecting or damaging treats. As ultra-low-power circuits and micro-systems develop, conventional batteries used for these systems could be replaced with smaller-sized and longer lifetime candidates. In this sense, energy harvesters hold great advantages such as unlimited lifetime, and no need for recharging or power cables.

In this project, students will conduct energy scavenging experiments with a photovoltaic module. The design process can be broke into the following steps:

1. The design and construction of a power electronic circuitry of the harvester;

2. The experimental measurements, data acquisition and analysis; and

3. The use of an energy harvesting circuitry to charge a battery and a super-capacitor bank.

\subsection{Solution}

The team decided to use solar energy to power the load because of its high energy density and availability. A large lithium phosphate battery provides and two super-capacitors are used as energy storage elements because of their many characteristics that suggest they can operate 10 years without maintenance. Also, a maximum power point tracking (MPPT) charge controller and a battery protection circuit allow the solar panel to charge the battery and to regulate the energy flow in the system. For the attachment method the team decided to clamp to the buoy or a platform used for monitoring. Wires run from the panel back under the bridge where the end node and the battery system are located. Wind load, fatigue, and stress calculations were performed to ensure the attachment was rugged enough to last 5 years.

\section{Project Description}

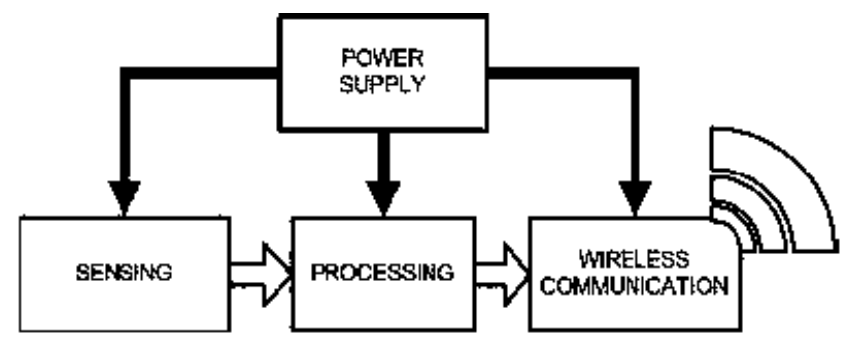

Figure 1. Block diagram of a sensor node

Sensing block may include one or more sensors. Among processing matches the sensor output to the digital processor, usually, a low-cost microcontroller. Commercial transceivers are used for wireless communications, usually transmitting in the free-licensed ISM band and can use a proprietary of standard (IEEE802.15.4) protocol. Power must be provided to different system stages. Nodes that only relay or receive data can skip functions such as sensing and analog processing. Transceivers are one of the most power consuming parts of a wireless node. The power of a transceiver in active mode is four to five orders of magnitude than in sleep mode ${ }^{12-14}$. 
However, power can also be dominated by the sensor stage ${ }^{14-19}$. Low-power sensors and electronic interfaces can help in the reduction of the power consumption of a WSN.

Average power consumption $\left(P_{\text {average }}\right)$ for a wireless sensor node ${ }^{6}$ is given by:

$$
P_{\text {average }}=D P_{\text {active }}+(1-D) P_{\text {sleep }}
$$

Here $D=t_{\text {active }} / T$ is the duty cycle, when the sensor node is operating, $P_{\text {active }}$ and $P_{\text {sleep }}$ are the power used by the WSN in active and passive mode, respectively, $T$ is the period. One way the lower $P_{\text {average }}$ is by reducing the duty cycle. The power supply stage must be able to provide both the total energy demanded during the expected lifetime and the instant power at the active time. Fig. 2 shows generic block diagram of a sensor node. Load accounts for sensing, processing and communication in Fig. $1^{12-15}$. Conversion efficiency of the system in Fig. 1 is defined as follows:

$$
\eta=\frac{P_{\text {Trnasferred }}}{P_{M P P}}
$$

Where $P_{M P P}$ is the power at the maximum power point (MPP) and P Transferred is the average power transferred to the energy buffer. When the super-capacitors are used, as is the proposed system, $P$ Transferred is usually computed as the value needed to increase the energy level from $E(t-T)$ to $E(t)$ during a given period $T$. Only harvester that always operates on the correct MPP can achieve efficiency over $90 \%$ and losses are only caused by the power dissipation of the system components. In our case, $\eta$ has been computed considering the DC-DC converter intrinsic losses.

\subsection{System Architecture and Design}

Typical design issues for solar harvesters are: the PV module size limiting the maximum power budget; the power consumed by the MPPT and control circuits (usually taking large part of the harvested energy), and the load and the powered systems that could interact with the harvester ${ }^{19}$ ${ }^{21}$. The harvester architecture, designed after several simulation steps with the proposed model for the PV modules, is shown in Fig. 2. The architecture of the developed harvester consists of three main subsystems: a) the maximum power point tracker sense the light intensity and control the buffer stage forcing the PV module to work in most efficient conditions; b) buffer stage (BS) stores the energy collected form the solar panel into the energy storage devices (super-capacitor and battery); and c) output stage (OS) generates a stable voltage supply for the low-power WSN applications. In the following we introduce the building blocks providing design guidelines and considerations, which help to optimize the performance of the harvester.

The MPPT algorithm is designed to automatically find the operating point (VPV, IPV) at which a PV module should operate to obtain the maximum output power under a given temperature and irradiance, following is when the light intensity changes. There are several algorithms to the track the maximum power point (MPP) voltage ${ }^{14-18}$. The most popular ones are Perturb and Observe (P\&O) and Fractional Open-Current Voltage (FOCV) ${ }^{20,21}$, which was adopted in this project. The P\&O method requires complex control is suitable WSN applications. The shortcomings of this method are the high cost and complexity. On the other hand, FOCV is largely 
used in small-scale and low-power PV applications. This method exploits the nearly linear relationship between PV module operating voltage at MPP $\mathrm{V}_{\mathrm{MP}}$ and its open-circuit voltage $\mathrm{V}_{\text {oc }}$ :

$$
V_{M p p} \cong K_{F O C} \cdot V_{O C}
$$

$\mathrm{K}_{\mathrm{FOC}}$ is a constant that ranges from 0.71 to 0.78 , depending slightly on irradiance conditions ${ }^{14}$. Since the linear factor depends on the characteristics of the solar cell that is being used, it usually has to be computed in advance by empirically or experimentally determining $\mathrm{V}_{\mathrm{MPP}}$ and $\mathrm{V}_{\mathrm{OC}}$ for a specific solar cell at different irradiance and temperature levels. However, this is only an approximation and a solar cell rarely operates in its exact MPP, but this MPPT technique is easy and inexpensive to implement and most important does not require a microcontroller, uses lower power to operate. The MPP can be approximated measuring periodically $\mathrm{V}_{\mathrm{oc}}$ by a temporary disconnection of the PV module from the circuit.



Fig. 2 Block diagram of the proposed system

The input stage accumulates energy collected from the PV panel into the super-capacitor and the battery. The input stage was designed as a DC-DC buck-boost converter, driven by a PWM signal generated by a controller to obtain a stable voltage. A $50 \mu \mathrm{F}$ that tolerates a $3.0 \mathrm{~V}$ maximum voltage was selected. Rechargeable batteries support a few hundred recharge cycles. For example, Lithium-Ion and NiMH supports at most 500 cycles. When submitted to frequent recharge cycles, like the solution in12-14, their lifetime are significantly degraded and the power module will not provide energy for a period of several years, as expected. Super-capacitors, also known as ultra-capacitors or double layer capacitors (DLC), are an emerging device for energy storage with a higher power density than batteries and 10 to 20 times that of regular electrolytic capacitors 14. They can be charged and discharged continuously without degradation and are ideal for pulsating applications, such as the typical WSN. Their disadvantage, compared to batteries, is a higher leak current, larger size and cost. Super-capacitors have superior characteristics over batteries in terms of cycle efficiency and cycle life ${ }^{13-16}$. Their cycle efficiency, defined as the ratio of the energy input to the energy output of an energy storage element, reaches almost $100 \%$. The terminal voltage of a super-capacitor (a linear function of its state of charge) is given by: 


$$
V_{C A P}(t)=\sqrt{\frac{2 \cdot E_{C A P}(t)}{C}}
$$

where $\mathrm{E}_{\mathrm{CAP}}$ is the energy stored in the super-capacitor, and $\mathrm{C}$ is the capacitance. $\mathrm{E}_{\mathrm{CAP}}$ increases or decreases dynamically as charge or discharge operations are performed. The same is true for $\mathrm{V}_{\mathrm{CAP}}$, whose variation is much higher than the terminal voltage of an ordinary battery, and therefore, the charger efficiency of a super-capacitor varies more significantly compared with that of a battery. A primary disadvantage of a super-capacitor is its larger self-discharge rate compared with that of an ordinary battery. A super-capacitor may lose more than $10 \%$ of its stored energy per day even if no load is connected to it.

The proposed solution uses a super-capacitor from PowerStor that present a very high energy density (100 times that of electrolyte caps) and power density (10 to 100 times that of batteries) ${ }^{14-16}$; equivalent series resistance (ESR) extremely low compared to activated carbon super-capacitors; relative low leak current; ample operating temperature range; and may be recharged hundred thousand times. Considering a duty cycle typical to WSN of $1 \%$, an active node current of $20 \mathrm{~mA}$ and a sleeping current of $5 \mu \mathrm{A}$, the average current is $0.01 \times 20 \mathrm{~mA}+$ $0.99 \times 5 \mu \mathrm{A}=0.205 \mathrm{~mA}$. The sensor node operating voltage is $2.80 \mathrm{~V}$ and the super-capacitors will power for 10 hours (estimated period without sun light). Two super-capacitors of $22 \mathrm{~F} / 2.5 \mathrm{~V}$ each in series are used in our design, providing the $2.80 \mathrm{~V}$ and minimizing the leak current.

Embedded systems, including WSNs contain advanced ICs that require a stable supply voltage to operate correctly and properly. This requirements demand a properly designed output stage with regulated voltage to deliver the power supply to the whole WSN node. A DC-DC regulator combined with a custom enabling and insulation circuits has been selected to maximize the output regulator efficiency. A Buck-Boost converter was selected to give more flexibility in providing a $3.3 \mathrm{~V}$ stable voltage with $\sim 90 \%$ efficiency. Note that efficiency depends also on super-capacitor, battery and the rest of the harvester components. Even with the same irradiance, usually there are significant changes in PV power (PPV) output due to various reasons. The MPPT methods discussed in previous subsections can be used to find the maximum $\mathrm{P}_{\mathrm{PV}}$ regardless of the irradiance.

The solar energy harvester is targeted to supply low-power WSN devices for marine monitoring under typical environmental conditions. Our scavenger is very flexible and can be attached to commercial sensor nodes which are not designed with energy harvesting features. Circuit consumption and losses vary with the current flowing through the power switches. The solar energy harvester is targeted to supply low-power WSN devices for environment monitoring under typical environmental conditions. He proposed scavenger is very flexible and can be attached to commercial sensor nodes which are not designed with energy harvesting features. Circuit consumption and losses vary with the current flowing through the power switches. In the most constraint conditions (low input power) the total power consumption is under $10 \mu \mathrm{W}$ making this architecture suitable for low power autonomous WSN. The power consumption for wireless sensor device provided by manufacturer with sensors and micro-controller in stand-by conditions is $<1 \mathrm{~mW}$, while the power consumption reaches $\sim 50 \mathrm{~mW}$ in active mode. Fig. 3 shows the experimental waveforms of the PV module voltage and current, and the output voltage 
and current for $1000 \mathrm{~W} / \mathrm{m}_{2}$ insolation. The PV voltage for both insolation values is kept at around $1.79 \mathrm{~V}$. However, the out power for the second insolation values is only about $60 \%$ of the previous one. The power difference is supplied by the storage unit of the harvester.

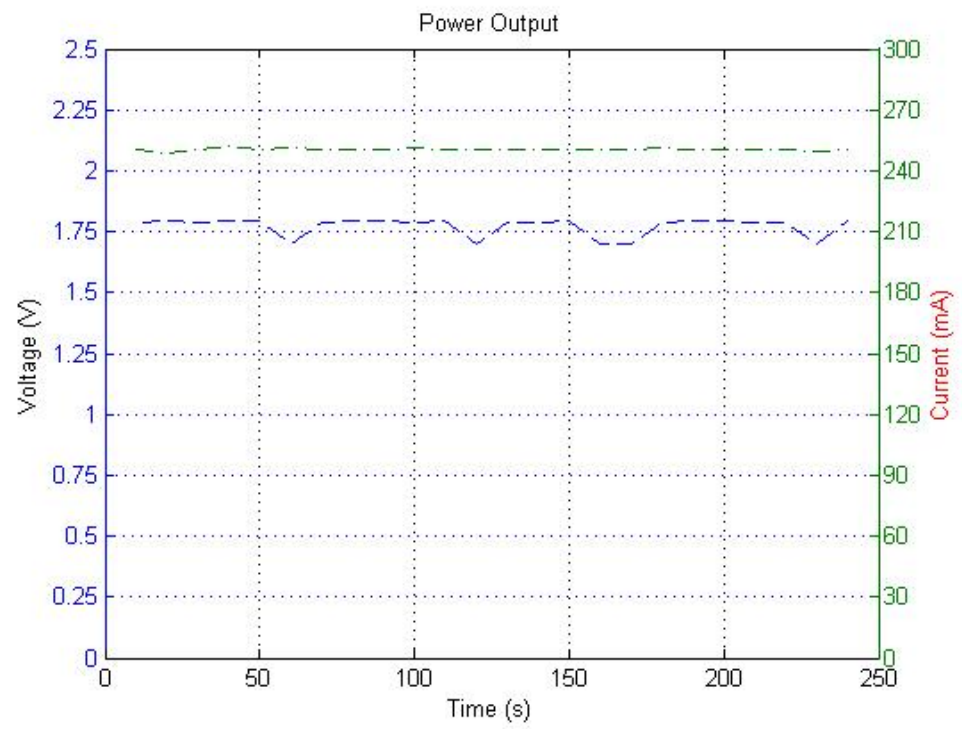

Figure 3 Waveforms showing PV voltage and PV current

\section{Final Remarks and Conclusions}

Students learn, verify, and reinforce theoretical concepts by performing experiments in the laboratory sessions and through the project experience. In our approach we adopted the principles of the problem-learning methodology. The design experience develops the students' lifelong learning skills, self-evaluations, self-discovery, and peer instruction in the design's creation, critique, and justification. Students learn to understand the manufacturer data sheets, application notes, and technical manuals. The experience, which would be difficult to complete individually, gives the students a sense of satisfaction and the accomplishment that is often lacking in many engineering courses, using traditional teaching approaches. Furthermore, the design experience motivates student learning and develops skills required in industry.

The development and implementation of a project solar energy harvesting in our senior project design course is described here. The project is used to allow students to apply fundamental engineering concepts as well as principles of engineering design. The societal impact of the project, Solar Energy Scavenging, also makes students more aware of what engineering can do to address current energy issues worldwide. Presently we are modifying the content of the project to address the main concern that many students expressed in their reflection papers, i.e. the level of complexity and the amount of time needed to complete the project. The project presented above, together with other projects proposed by the authors in the areas of renewable energy, energy harvesting and wireless sensor networks have been used to draw student's interest in the field of renewable energy sources, advanced and intelligent monitoring systems. 


\section{References}

1. R.S. Friedman, F.P. Deek, Innovation and education in the digital age: reconciling the roles of pedagogy, technology, and the business of learning,, IEEE Transactions on Engineering Management, Vol. 50, No. 4, Nov. 2003, pp. 403-412.

2. Wormley, D.N., "Challenges in Curriculum Renewal," International Journal Engineering Education, Vo. 20, No. 3, 2004, pp. 329-332.

3. Splitt, F., Environmentally Smart Engineering Education: A Brief on a Paradigm in Progress, Journal of Engineering Education, Vol. 91, 2002, pp. 447-450.

4. Blumenfeld, P.C., E. Soloway, R.W. Marx, J.S. Krajcik, M. Guzdial, and A. Palinscar, Motivating ProjectBased Learning: Sustaining the Doing, Supporting the Learning, Educ. Psychologist, Vol. 26, 1991, pp. 369-398. 5. A. Brobely and J. F. Kreider (editors), Distributed Generation the Power Paradigm of the New Millennium, CRC Press, 2001.

6. J. Peng, Survey on Key Technology of WSN-based Wetland Water Quality Remote Real-time Monitoring System, Chinese Journal of Sensors and Actuators, vol20, (1)2007,pp 183-186.

7. J. Peng, Research on Wireless Sensor Networks Routing Protocol for Water Environment Monitoring in Wetlands, International Conference on Innovative, Computing, Information and Control, 2006, pp 251-254. 8. Yang, H. Wu, H.; He, Y.: Architecture of wireless sensor network for monitoring aquatic environment of marine shellfish. In Proceedings of the 7th IEEE Asian Control Conference, Hong Kong, August 2009, pp. 1147-1151.

9. Cooper, H. L. (2006), Undergraduate renewable energy projects to support energy solutions of the future. Proceedings of the 2006 ASME International Mechanical Engineering Conference (IMECE2006), pp. 491-495 10. Engineering Accreditation Commission, Criteria for Accrediting Engineering Programs, http://www.abet.org/criteria.html. (2002).

11. Felder, R.M., and R. Brent, "Designing and Teaching Courses to Satisfy the ABET Engineering Criteria," Journal of Engineering Education, Vol. 92, No. 1, 2003, pp. 7-25.

12. S. Sudevalayam and P. Kulkarni, "Energy Harvesting Sensor Nodes: Survey and Implications," IEEE Commun. Surveys Tuts., 2011.

13. Z. A. Eu, H.-P. Tan, and W. K. G. Seah, "Wireless Sensor Networks Powered by Ambient Energy Harvesting: An Empirical Characterization," in IEEE International Conference on Communications (ICC), 2010.

14. F. Simjee and P. H. Chou, "Everlast: long-life, super-capacitor-operated wireless sensor node," in ISLPED '06: Proceedings of the 2006 international symposium on Low power electronics and design, (New York, NY, USA), pp. 197-202, ACM Press, 2006.

15. L. Zubieta and R. Bonert, "Characterization of Double-Layer Capacitors (DLCs) for Power Electronics Applications," in Industry Applications Conference, 1998. Thirty-Third IAS Annual Meeting. The 1998 IEEE, vol. 2, pp. 1149-1154, 1998..

16. PowerStor Supercapacitors, "Powerstor Supercapacitors datasheet," Available on: http://www.cooperbussmann.com/, 2008.

17. D. Brunelli, L. Benini, C. Moser, and L. Thiele, "An efficient solar energy harvester for wireless sensor nodes", DATE, pp 104-109, 2008.

18. A. B. da Cunha and D. C. Da Silva, "Energy-Efficient Characterization of Solar Panel-Supercapacitors Systems for Energy-Harvesting Aware Wireless Sensor Nodes", in: Proc. 2009 IEEE 20th International Symposium on Personal, Indoor and Mobile Radio Communications, Tokyo, Japan, pp. 2275-2279, 13-16 Sept. 2009.

19. F. Simjee and P. H. Chou, "Everlast: long-life, supercapacitor-operated wireless sensor node," in ISLPED '06: Proceedings of the 2006 international symposium on Low power electronics and design, (New York, NY, USA), pp. 197-202, ACM Press, 2006.

20. A. Pandey, N. Dasguta, and A. K. Murkerjee, “A simple single-sensor MPPT solution”, IEEE Transaction on Power Electronics, vol.22, no.2, pp 698-700, 2007.

21. R. Belu, "Power Electronics and Controls for Photovoltaics" in Handbook of Research on Solar Energy Systems and Technologies (Eds: Dr. Sohail Anwar and Dr. Harry Efstathiadis),2011 (to be published 